\title{
FURTHER EXTENDING A COMPLETE CONVEX METRIC
}

\author{
ROBERT A. DOOLEY 1
}

\begin{abstract}
A metric $D$ is convex if for every two points $x, z$ there is a third point $y$ such that $D(x, y)+D(y, z)=D(x, z)$. A generalized continuum is a connected, locally compact, metric space. Let $M_{1}$ be a nonempty space with a complete convex metric $D_{1}$ and let $M_{2}$ be a nonempty locally connected generalized continuum. The following condition is shown to be necessary and sufficient for there to exist a complete convex metric for $M_{1} \cup M_{2}$ that extends $D_{1}: M_{1} \cap M_{2}$ is a nonempty subspace of both $M_{1}$ and $M_{2}$ which is closed in $M_{2}$ and whose $M_{2}$ boundary is closed in $M_{1}$.
\end{abstract}

1. Introduction. In this paper we continue the investigation of a previous article [5], regarding the extendability of complete convex metrics. In [5] basic definitions are provided, and a sufficient condition is obtained for the extension of a given complete convex metric across a locally connected generalized continuum. The aim of the present paper is to weaken that sufficient condition so that it is also necessary, as follows: if $M_{1}$ is a space with a complete convex metric $D_{1}$ and $M_{2}$ is a locally connected generalized continuum, a necessary and sufficient condition that $D_{1}$ can be extended to a complete convex metric for $M_{1} \cup M_{2}$ is that $M_{1} \cap M_{2}$ be a nonempty subspace of both $M_{1}$ and $M_{2}$ which is closed in $M_{2}$ and whose $M_{2}$ boundary is closed in $M_{1}$. The following conventions, in addition to those to be mentioned in $\S 2$, will be observed throughout this paper. All given topological spaces are assumed to be nonempty. If $D$ is a metric for a space $M$, we will write $D(p ; \varepsilon)$ to denote the open ball $\{x: D(p, x)<\varepsilon\}$ and $\bar{D}(p ; \varepsilon)$ for the closed ball $\{x: D(p, x) \leqq \varepsilon\}$.

2. The union topology. The union of two topological spaces $M_{1}$ and $M_{2}$ whose topologies agree on their intersection will in this paper be considered to have a certain natural topology, namely the collection $\tau_{0}$ of all

Presented to the Society, March 20, 1972; received by the editors September 29, 1972 and, in revised form, December 26, 1972.

AMS (MOS) subject classifications (1970). Primary 54E50; Secondary 54F15.

Key words and phrases. Convex metric, generalized continuum.

${ }^{1}$ This research was begun with the support of NSF Grant GZ-1694 and comprises a part of the author's dissertation at Oklahoma State University under the direction of Professor John M. Jobe. 
sets $Q$ in $M_{1} \cup M_{2}$ such that $Q \cap M_{i}$ is open in $M_{i}$ for $i=1,2$. Seen otherwise, $\tau_{0}$ is the largest topology on $M_{1} \cup M_{2}$ for which $M_{1}$ and $M_{2}$ are subspaces. For the spaces $M_{1}$ and $M_{2}$ in the propositions of this section, we assume that their topologies do agree on their intersection and that $\tau_{0}$ has the meaning described above. The simple proofs of these propositions are omitted. The claim of $\tau_{0}$ to naturalness, at least for the purposes of this paper, is given by $(2.1)$.

Proposition 2.1. If $M_{1} \cap M_{2}$ is $M_{2}$ closed, and if the $M_{2}$ boundary of $M_{1} \cap M_{2}$ is $M_{1}$ closed, then $M_{1} \backslash M_{2}$ and $M_{2} \backslash M_{1}$ are $\tau_{0}$ separated sets.

Proposition 2.2. If $M_{1} \backslash M_{2}$ and $M_{2} \mid M_{1}$ are separated sets in some topology $\tau$ on $M_{1} \cup M_{2}$ for which $M_{1}$ and $M_{2}$ are subspaces, then $\tau=\tau_{0}$.

Proposition 2.3. If $R_{i}$ is a $M_{i}$ neighborhood of a point $x$ for $i=1,2$, then $x$ is $\tau_{0}$ interior to $R_{1} \cup R_{2}$.

Proposition 2.4. If $M_{1}$ and $M_{2}$ are locally compact spaces such that $M_{1} \cap M_{2}$ is closed in $M_{2}$ and the $M_{2}$ boundary of $M_{1} \cap M_{2}$ is closed in $M_{1}$, and if $M_{1} \cup M_{2}$ is Hausdorff, then $M_{1} \cup M_{2}$ is locally compact.

An example of the union of two locally connected generalized continua in the plane sheds light on both (2.4) and (2.5). If $M_{1}=E^{1} \times(-\infty, 0]$ and $M_{2}=(-\infty, 0) \times E^{1}$, we observe that the origin has neither a conditionally compact neighborhood nor a countable local base with respect to $\tau_{0}$.

PROPOSITION 2.5. If $M_{1} \cup M_{2}$ is both Hausdorff and first countable, then $B \cap M_{1}$ is $M_{1}$ closed, where $B$ is the $M_{2}$ boundary of $M_{1} \cap M_{2}$.

Proposition 2.6. Let $M_{1}$ be a space with a complete metric $D_{1}$ and let $M_{2}$ be any topological space. In order that $M_{1} \cup M_{2}$ be a connected space and admit some metric $D_{3}$ extending $D_{1}$, it is necessary that $M_{1} \cap M_{2}$ be a nonempty subspace of both $M_{1}$ and $M_{2}$ which is closed in $M_{2}$ and that the $M_{2}$ boundary of $M_{1} \cap M_{2}$ be closed in $M_{1}$.

3. Segmented convex metrics. A metric is segmented convex if each pair of its points are joined by at least one segment. It is clear that the segmented convex metrics occupy an intermediate position between the convex and the complete convex metrics, in that every segmented convex metric is convex and, by a well-known theorem of Menger [8], every complete convex metric is segmented convex. The rationals in $E^{1}$ with the euclidean metric form a convex metric space that does not admit a segmented convex metric. Similarly, not every space that admits a segmented convex metric must admit a complete convex metric; indeed, we can embed a metric space of first category isometrically as a closed subset of a 
normed linear space, which cannot therefore be topologically complete [1]. However, we observe the following characterization.

THEOREM 3.1. If $M$ is a locally compact space, the following statements are equivalent:

(i) $M$ is a locally connected generalized continuum.

(ii) $M$ admits a complete convex metric.

(iii) $M$ admits a segmented convex metric.

Proof. The proofs for (i) $\Rightarrow$ (ii) and for (ii) $\Rightarrow$ (iii) are given in [9] and [8] respectively. For (iii) $\Rightarrow$ (i), the admission of a segmented convex metric implies that $M$ is connected and locally connected, since open balls are connected. Thus, $M$ is a locally connected generalized continuum.

In this paper, use is made of segmented convex metrics through the following theorem.

THEOREM 3.2. In a locally compact space with a segmented convex metric, every compact metric ball is a Peano continuum.

Proof. The proof follows the general scheme of a proof in Hall and Spencer [6, Theorem V. 6.23].

COROLlaRY 3.3. In a locally compact space with a complete convex metric, every closed metric ball is a Peano continuum.

Proof. Every closed metric ball is compact [7].

4. Extension theorems. The following theorem is a stronger statement of Theorem 1 of [5].

Theorem 4.1. Let $M_{1}$ be a space with complete convex metric $D_{1}$ and let $M_{2}$ be a locally connected generalized continuum with complete convex metric $D_{2}$, whose intersection with $M_{1}$ is a nonempty, compact subspace of both $M_{1}$ and $M_{2}$. Then for any $\varepsilon>0$ and for any two nonempty subsets $C$ and $H$ of $M_{2}$ with $D_{2}\left(C, H \cup\left(M_{1} \cap M_{2}\right)\right)>0$, there is a complete convex metric $D_{3}$ for $M_{1} \cup M_{2}$ that extends $D_{1}$, satisfies $D_{3}(C, H) \geqq \varepsilon$, and has the property that if $D_{3}(x, y)<D_{2}(x, y)$ for two points $x, y$ of $M_{2} \backslash M_{1}$, then $x$ and $y$ have $a$ $D_{3}$ between points in $M_{1}$.

Proof. Let $\delta=D_{2}\left(C, H \cup\left(M_{1} \cap M_{2}\right)\right)$. The proof of Theorem 1 in [5], following the construction of Bing [2], now suffices for the present theorem, if the initial function $F(x)$ in that proof is chosen to have a first derivative which always exceeds both 1 and $\varepsilon / \delta$. For use in the proof of (4.3), we note here that the function $D_{0}(x, y)$ defined in [5] is never less than either $D_{2}(x, y)$ or $(\varepsilon / \delta) D_{2}(x, y)$. From this fact and the definition of the metric $D_{3}$ given in [5], the desired properties of $D_{3}$ readily follow. 
Given a complete convex metric for a locally compact space, not every compact set need be contained in a compact set on which the metric is convex; in fact, there is a noncompact generalized continuum $X$ in the plane that contains three points and has a complete convex metric which is not convex on any closed proper subset of $X$ containing those three points [4]. The following theorem shows, however, that a locally connected generalized continuum can be remetrized with a complete convex metric for which the property in question will in fact hold.

THEOREM 4.2. Let $M$ be a locally connected generalized continuum with complete convex metric $D$. Given any point $p$ of $M$, there is a complete convex metric $E$ for $M$ that is convex on $\bar{D}(p ; n)$ and has the property that if $D(p, x)=n$ then $E\left(x, D\left(p ; n-\frac{1}{2}\right)\right) \geqq 1$, for $n=1,2, \cdots$.

Proof. For each $n$, we see from (3.3) that $P_{n}=\bar{D}(p ; n)$ is a Peano continuum; moreover, the two sets $C_{n}=\{x: D(p, x)=n\}$ and $H_{n-1}=$ $\left\{x: D(p, x)=n-\frac{1}{2}\right\}$ are compact and disjoint. By (4.1) there is a convex metric $E_{1}$ for $P_{1}$ such that $E_{1}\left(C_{1}, H_{0}\right) \geqq 1$ if $C_{1} \neq \varnothing$. By repeated use of (4.1), a sequence $E_{1}, E_{2}, \cdots$ of convex metrics respectively for $P_{1}, P_{2}, \cdots$ may be defined inductively so that $E_{n+1}$ extends $E_{n}$ and $E_{n}\left(C_{n}, H_{n-1}\right) \geqq 1$ whenever $C_{n} \neq \varnothing$. If $E$ is the union of all these metrics $E_{n}$, then $E$ is a segmented convex metric for the space $M$. Moreover, since $E\left(C_{n}, p\right) \geqq n$ as long as $C_{n} \neq \varnothing$ and consequently every $E$ bounded set is also $D$ bounded, then $E$ is complete.

THEOREM 4.3. Let $M_{1}$ be a space with a complete convex metric $D_{1}$ and let $M_{2}$ be a locally connected generalized continuum. In order for there to be a complete convex metric for $M_{1} \cup M_{2}$ that extends $D_{1}$, it is necessary and sufficient that $M_{1} \cap M_{2}$ be a nonempty subspace of both $M_{1}$ and $M_{2}$ which is closed in $M_{2}$ and that the $M_{2}$ boundary of $M_{1} \cap M_{2}$ be closed in $M_{1}$.

Proof. Necessity is given by (2.6). For the proof of sufficiency, let $p$ be in $M_{1} \cap M_{2}$ and let $D$ be any complete convex metric for $M_{2}$. By (4.2) there is a complete convex metric $D_{2}$ for $M_{2}$ whose restriction $D_{2}^{n}$ to $P_{n}=$ $\bar{D}(p ; n)$ is convex, and which has the property that if $D(p, x)=n$ then $D_{2}\left(x, \bar{D}\left(p ; n-\frac{1}{2}\right)\right) \geqq 1$, for $n=1,2, \cdots$.

Since $M_{1} \cap M_{2}$ is closed in $M_{2}$, then $M_{1} \cap P_{1}$ is compact. Hence, (4.1) may be applied by replacing $M_{1}, D_{1}, M_{2}$, and $D_{2}$ by $M_{1}, D_{1}, P_{1}$, and $D_{2}^{1}$ respectively in the hypothesis; let $D_{0}^{1}$ and $D_{3}^{1}$ be the $D_{0}$ and $D_{3}$ given respectively by the proof and conclusion. (The sets $C$ and $H$ in (4.1) will not be used here.) We note that $D_{0}^{1}(u, v) \geqq D_{2}^{1}(u, v)=D_{2}(u, v)$ whenever $D_{0}^{1}(u, v)$ is defined, and that if $x$ lies in $P_{1} \backslash M_{1}$ and $y$ in $M_{1}, D_{3}^{1}(x, y)$ is defined to be the infimum of sums $D_{0}^{1}(x, a)+D_{1}(a, y)$ for certain points $a$ in the $P_{1}$ boundary of $M_{1} \cap P_{1}$. 
Proceeding inductively, suppose that $D_{3}^{n}$ is a complete convex metric for $M_{1} \cup P_{n}$ which extends $D_{1}$. Again apply (4.1) by replacing $M_{1}, D_{1}, M_{2}$, and $D_{2}$ by $M_{1} \cup P_{n}, D_{3}^{n}, P_{n+1}$, and $D_{2}^{n+1}$ respectively, and obtain $D_{0}^{n+1}$ and $D_{3}^{n+1}$ in place of $D_{0}$ and $D_{3}$. We have that $D_{3}^{n+1}$ is a complete convex metric for $M_{1} \cup P_{n}$ that extends $D_{3}^{n}$, with the property that whenever $D_{3}^{n+1}(x, y)<$ $D_{2}^{n+1}(x, y)$ for points $x, y$ of $P_{n+1} \backslash\left(M_{1} \cup P_{n}\right)$, then $x$ and $y$ have a $D_{3}^{n+1}$ between point in $M_{1} \cup P_{n}$. Again, we should note that $D_{0}^{n+1}(u, v) \geqq$ $D_{2}^{n+1}(u, v)=D_{2}(u, v)$ whenever $D_{0}^{n+1}(u, v)$ is defined; moreover, for points $x$ in $P_{n+1} \backslash\left(M_{1} \cup P_{n}\right)$ and $y$ in $M_{1} \cup P_{n}$, the value $D_{3}^{n+1}(x, y)$ is defined to be an infimum of sums $D_{0}^{n+1}(x, a)+D_{3}^{n}(a, y)$ for certain points $a$ in the $P_{n+1}$ boundary of $\left(M_{1} \cup P_{n}\right) \cap P_{n+1}$.

Define $D_{3}$ as the union of the metrics $D_{3}^{n}$, and for convenience let $P_{0}=$ $M_{1} \cap P_{1}$ and $D_{3}^{0}=D_{1}$. It is immediate that $D_{3}$ is a segmented convex metric which extends $D_{1}$. Assertions (iv) and (vi) below complete the proof.

(i) For points $x$ in $P_{n}, y$ in $M_{1}$, and for $\eta>0$, there is a point $z$ in $M_{1} \cap P_{n}$ such that $D_{3}^{n}(x, y)+\eta>D_{2}(x, z)+D_{1}(z, y)$. If $x$ is not in $M_{1}$, then $z$ can be chosen in the $P_{n}$ boundary of $M_{1} \cap P_{n}$.

(ii) For points $x$ in $P_{n+k}, y$ in $M_{1} \cup P_{k}(k=0,1, \cdots ; n=1,2, \cdots)$, and for $\eta>0$, there is a point $z$ in $\left(M_{1} \cup P_{k}\right) \cap P_{n+k}$ such that $D_{3}^{n+k}(x, y)+\eta>$ $D_{2}(x, z)+D_{3}^{k}(z, y)$.

(iii) $D_{2}$ is topologically equivalent to $D_{3}$ restricted to $M_{2}$.

(iv) $D_{3}$ is a metric for $M_{1} \cup M_{2}$.

(v) If point $t$ is in $P_{n}$ and $v$ is in $M_{2} \mid P_{n+1}$ for some $n>0$, there is a $D_{3}$ between point $u$ of $t$ and $v$ such that $D(p, u)=n+\frac{1}{2}$ and $P_{n}$ contains no $D_{3}$ between point of $u$ and $v$.

(vi) $D_{3}$ is complete.

For (i) let $x$ in $P_{n}, y$ in $M_{1}$, and $\eta>0$ be given. If $x$ is in $M_{1}$, then $x$ itself may be taken for $z$ since $D_{3}^{n}(x, y)=D_{1}(x, y)$. Therefore, with the assumption that $x$ is not in $M_{1}$, the proof of (i) is given by induction on $n$. If $x$ is in $P_{1} \backslash M_{1}$, then by the definition of $D_{3}^{1}(x, y)$ there is a point $z$ on the $P_{1}$ boundary of $M_{1} \cap P_{1}$ such that $D_{3}^{1}(x, y)+\eta>D_{0}^{1}(x, z)+D_{1}(z, y) \geqq$ $D_{2}(x, z)+D_{1}(z, y)$. Proceeding inductively, assume that (ii) holds for $n=k$ and arbitrary $\eta^{\prime}>0$, and let $x \in P_{k+1} \backslash\left(M_{1} \cup P_{k}\right)$ with $\eta>0$. From the definition of $D_{3}^{k+1}(x, y)$ there is a point $z^{\prime}$ on the $P_{k+1}$ boundary of $\left(M_{1} \cup P_{k}\right) \cap P_{k+1}$ such that

$$
D_{3}^{k+1}(x, y)+\eta / 2>D_{0}^{k+1}\left(x, z^{\prime}\right)+D_{3}^{k}\left(z^{\prime}, y\right) \geqq D_{2}\left(x, z^{\prime}\right)+D_{3}^{k}\left(z^{\prime}, y\right) .
$$

If $z^{\prime}$ is in $M_{1} \cap P_{k+1}$, then $z^{\prime}$ is on the $P_{k+1}$ boundary of $M_{1} \cap P_{k+1}$, and since $D_{3}^{k}\left(z^{\prime}, y\right)=D_{1}\left(z^{\prime}, y\right)$, inequality (1) shows that $z=z^{\prime}$ satisfies (i). If however $z^{\prime}$ is not in $M_{1}$, then $z^{\prime}$ is in $P_{k}$. Thus by the induction hypothesis for the points $z^{\prime}$ and $y$, there is a point $z$ on the $P_{k}$ boundary of $M_{1} \cap P_{k}$, hence on 
the $P_{k+1}$ boundary of $M_{1} \cap P_{k+1}$, such that

$$
D_{3}^{k}\left(z^{\prime}, y\right)+\eta / 2>D_{2}\left(z^{\prime}, z\right)+D_{1}(z, y) \text {. }
$$

Upon combining this inequality with (1) and the triangle inequality, we arrive at the desired inequality in $x, y$, and $z$, completing the induction.

Assertion (ii) can be proved by double induction by using (i) as the initialization $k=0$ and an argument similar to the proof of (i) to complete the induction.

To prove for (iii) that $D_{2}$ and $D_{3}$ give the same topology on $M_{2}$, first let $D_{3}(x ; \varepsilon)$ be given with $x$ a point of $M_{2}$. Since by [7] the set $\bar{D}_{2}(x ; \varepsilon)$ is compact, it lies in $P_{n}$ for some $n$. But since $D_{3}^{n}(x ; \varepsilon) \cap M_{2}$ is $D_{2}^{n}$ open, there is some $\varepsilon \geqq \delta>0$ such that $D_{2}^{n}(x ; \delta) \subset D_{3}^{n}(x ; \varepsilon) \cap M_{2}$. Since $D_{2}(x ; \delta) \subset P_{n}$, then $D_{2}(x ; \delta)=D_{2}^{n}(x ; \delta) \subset D_{3}(x ; \varepsilon)$.

Now let $D_{2}(x ; \varepsilon)$ be an arbitrary $D_{2}$ ball, and first suppose that $x$ is in $M_{1} \cap M_{2}$. Then there is some $\varepsilon / 2 \geqq \delta>0$ such that $D_{1}(x ; \delta) \cap M_{2} \subset$ $D_{2}(x ; \varepsilon / 2) \cap M_{1}$. For any point $y$ of $D_{3}(x ; \delta) \cap M_{2}$, there is by (i) some point $z$ in $M_{1} \cap M_{2}$ such that $\delta>D_{2}(y, z)+D_{1}(z, x)$. Since $z$ is thus in $D_{1}(x ; \delta) \cap M_{2}$, then $D_{2}(x, z)<\varepsilon / 2$ and the triangle inequality shows that $y$ is in $D_{2}(x ; \varepsilon)$. Hence in this case, $D_{3}(x ; \delta) \cap M_{2} \subset D_{2}(x ; \varepsilon)$. If instead $x$ is in $M_{2} \backslash M_{1}$, there is some $\varepsilon / 2 \geqq \mu>0$ such that the compact set $\bar{D}_{2}(x ; \mu)$ is in $M_{2} \backslash M_{1}$ and in some $P_{n}$, so that $D_{2}(x ; \mu)=D_{2}^{n}(x ; \mu)$. Since $D_{2}^{n}$ and $D_{3}^{n}$ are equivalent on $P_{n}$, there is some $\mu \geqq v>0$ for which $D_{3}^{n}(x ; v) \cap M_{2} \subset$ $D_{2}(x ; \mu)$. Since any point $y$ of $D_{3}(x ; v) \cap M_{2}$ lies in $P_{n+k}$ for some $k \geqq 1$, by (ii) there is a point $z$ of $\left(M_{1} \cup P_{n}\right) \cap P_{n+k}$ satisfying $\nu>D_{2}(y, z)+$ $D_{3}^{n}(z, x)$. Thus $z$ lies in $D_{3}^{n}(x ; v) \cap M_{2}$ and hence in $D_{2}(x ; \mu)$, so that as above the triangle inequality places $y$ in $D_{2}(x ; \varepsilon)$. Therefore $D_{3}(x ; \nu) \cap$ $M_{2} \subset D_{2}(x ; \varepsilon)$, and (iii) is established.

Since $M_{1}$ and by (iii) also $M_{2}$ are subspaces of $\left(M_{1} \cup M_{2}, D_{3}\right)$, statement (iv) follows from (2.2) as we note, using (i), that $M_{1} \backslash M_{2}$ and $M_{2} \backslash M_{1}$ are separated sets in $\left(M_{1} \cup M_{2}, D_{3}\right)$.

If $(v)$ were false, there would exist sequences of points $\left\{t_{i}\right\}$ and $\left\{u_{i}\right\}$, satisfying $t_{i} \in P_{n}$ and $D_{3}\left(p, u_{i}\right)=n+\frac{1}{2}$, such that $\lim D_{3}\left(t_{i}, u_{i}\right)=0$. But since $P_{n}$ and $\left\{x: D_{3}(p, x)=n+\frac{1}{2}\right\}$ are disjoint compact sets, this is impossible.

To show for (vi) that $D_{3}$ is complete, let $\left\{x_{k}\right\}$ be a $D_{3}$ Cauchy sequence. It may be assumed that $\left\{x_{k}\right\}$ lies entirely in $M_{2} \backslash M_{1}$ and has no subsequence that lies entirely in one of the sets $P_{n}$. In fact, if $x_{k}$ lies in $P_{n_{k}} \backslash P_{n_{k}-1}$ for each $k$, it may be assumed that $n_{k}+1<n_{k+1}$. Suppose that for only a finite set (here assumed empty) of indices $k$ the points $x_{k}$ and $x_{k+1}$ have a $D_{3}$ between point in $M_{1}$. Then, for each $k$, (v) shows that there is some $D_{3}$ between point $u$ of $x_{k}$ and $x_{k+1}$ such that $D(p, u)=n_{k}+\frac{1}{2}$ and $P_{n_{k}}$ contains no $D_{3}$ between point of $u$ and $x_{k+1}$. Since $x_{k+1}$ lies outside $P_{n_{k}+1}$, there is a 
$D_{3}$ between point $v$ of $u$ and $x_{k+1}$ satisfying $D(p, v)=n_{k}+1$, and moreover $P_{n_{k}}$ contains no $D_{3}$ between point of $u$ and $v$. Therefore, $u$ and $v$ can have no $D_{3}$ between point in $M_{1} \cup P_{n_{k}}$. Because of this fact and the construction of the metrics $D_{3}^{n_{k}+1}$ and $D_{2}$, it follows that $D_{3}\left(x_{k}, x_{k+1}\right) \geqq D_{3}(u, v)=$ $D_{3}^{n_{k}+1}(u, v) \geqq D_{2}^{n_{k}+1}(u, v)=D_{2}(u, v) \geqq 1$, and $\left\{x_{k}\right\}$ cannot be $D_{3}$ Cauchy.

Hence, there must be a subsequence $\left\{x_{k_{i}}\right\}$ of $\left\{x_{k}\right\}$ for which the points $x_{k_{i}}$ and $x_{k_{i}+1}$ have a $D_{3}$ between point $y_{i}$ in $M_{1}$. Then $\left\{y_{i}\right\}$ is a $D_{1}$ Cauchy sequence that converges to some point $y$ in $M_{1}$, and it follows that $\left\{x_{k}\right\}$ also converges to $y$. Therefore, $D_{3}$ is complete.

COROLLARY 4.4 If a closed subspace $M_{1}$ of a locally connected generalized continuum $M_{2}$ has a complete convex metric $D_{1}$, then $D_{1}$ can be extended to a complete convex metric for $M_{2}$.

The above corollary is analogous to Bing's classic extension theorem for arbitrary metric spaces [3]. The following theorem now follows easily from (2.4), (3.1), and (4.3).

THEOREM 4.5. Let $M_{1}$ and $M_{2}$ be locally connected generalized continua. In order for $M_{1} \cup M_{2}$ to be a locally connected generalized continuum and for every complete convex metric for $M_{1}$ to extend to a complete convex metric for $M_{1} \cup M_{2}$, it is necessary and sufficient that $M_{1} \cap M_{2}$ be a nonempty subspace of both $M_{1}$ and $M_{2}$ which is closed in $M_{2}$ and that the $M_{2}$ boundary of $M_{1} \cap M_{2}$ be closed in $M_{1}$.

\section{REFERENCES}

1. R. F. Arens and J. Eells, Jr., On embedding uniform and topological spaces, Pacific J. Math. 6 (1956), 397-403. MR 18, 406.

2. R. H. Bing, A convex metric for a locally connected continuum, Bull. Amer. Math. Soc. 55 (1949), 812-819. MR 11, 194.

3. — Extending a metric, Duke Math. J. 14 (1947), 511-519. MR 9, 521.

4. R. A. Dooley, Complete convex metrics for generalized continua, Ph.D. Dissertation, Oklahoma State University, 1972.

5. - Extending a complete convex metric, Proc. Amer. Math. Soc. 34 (1972), 553-559.

6. D. W. Hall and G. L. Spencer II, Elementary topology, Wiley, New York, 1955. MR 17, 649.

7. A. Lelek and J. Mycielski, On convex metric spaces. IV, Fund. Math. 61 (1967), 171-176. MR 36 \#4520.

8. K. Menger, Untersuchungen über allgemeine Metrik, Math. Ann. 100 (1928), 75163.

9. A. Tominaga and T. Tanaka, Convexification of locally connected generalized continua, J. Sci. Hiroshima Univ. Ser. A 19 (1955), 301-306. MR 17, 1230.

Department of Mathematics, Oklahoma State University, Stillwater, OklaHOMA 74074 